OPEN ACCESS

Edited by:

Christos Papadelis, Cook Children's Medical Center, United States

Reviewed by:

Claudio Luis Ferre, Burke Neurological Institute (BNI), United States

Yanlong Song,

University of Texas at Arlington, United States Hsing-Ching Cherie Kuo, UC Davis Medical Center, United States

*Correspondence: Eileen G. Fowler efowler@mednet.ucla.edu

Specialty section: This article was submitted to Motor Neuroscience, a section of the journal Frontiers in Human Neuroscience

Received: 08 February 2020 Accepted: 14 April 2020 Published: 25 May 2020

Citation: Fowler EG, Oppenheim WL, Greenberg MB, Staudt LA, Joshi SH and Silverman DHS (2020) Brain Metabolism During a Lower Extremity Voluntary Movement Task in Children with Spastic Cerebral Palsy. Front. Hum. Neurosci. 14:159. doi: 10.3389/fnhum.2020.00159

\section{Brain Metabolism During A Lower Extremity Voluntary Movement Task in Children With Spastic Cerebral Palsy}

\author{
Eileen G. Fowler ${ }^{1,2 *}$, William L. Oppenheim ${ }^{1}$, Marcia B. Greenberg ${ }^{1}$, Loretta A. Staudt ${ }^{1}$, \\ Shantanu H. Joshi ${ }^{3,4}$ and Daniel H. S. Silverman ${ }^{5,6}$ \\ ${ }^{1}$ Center for Cerebral Palsy, Department of Orthopaedic Surgery, University of California, Los Angeles, Los Angeles, CA, \\ United States, ${ }^{2}$ Tarjan Center at UCLA, Los Angeles, CA, United States, ${ }^{3}$ Department of Neurology, University of California, \\ Los Angeles, Los Angeles, CA, United States, ${ }^{4}$ Department of Bioengineering, University of California, Los Angeles, \\ Los Angeles, CA, United States, ${ }^{5}$ Department of Molecular and Medical Pharmacology, University of California, Los Angeles, \\ Los Angeles, CA, United States, ${ }^{6}$ Ahmanson Translational Imaging Division, UCLA Health System, Los Angeles, CA, \\ United States
}

Reduced selective voluntary motor control (SVMC) is a primary impairment due to corticospinal tract (CST) injury in spastic cerebral palsy (CP). There are few studies of brain metabolism in $\mathrm{CP}$ and none have examined brain metabolism during a motor task. Nine children with bilateral spastic CP [Age: 6-11 years, Gross Motor Function Classification System (GMFCS) Levels II-V] completed this study. SVMC was evaluated using Selective Control Assessment of the Lower Extremity (SCALE) ranging from 0 (absent) to 10 (normal). Brain metabolism was measured using positron emission tomography (PET) scanning in association with a selective ankle motor task. Whole brain activation maps as well as $\mathrm{ROI}$ averaged metabolic activity were correlated with SCALE scores. The contralateral sensorimotor and superior parietal cortex were positively correlated with SCALE scores $(p<0.0005)$. In contrast, a negative correlation of metabolic activity with SCALE was found in the cerebellum $(p<0.0005)$. Subsequent $\mathrm{ROI}$ analysis showed that both ipsilateral and contralateral cerebellar metabolism correlated with SCALE but the relationship for the ipsilateral cerebellum was stronger $\left(R^{2}=0.80, p<0.001\right.$ vs. $\left.R^{2}=0.46, p=0.045\right)$. Decreased cortical and increased cerebellar activation in children with less SVMC may be related to task difficulty, activation of new motor learning paradigms in the cerebellum and potential engagement of alternative motor systems when CSTs are focally damaged. These results support SCALE as a clinical correlate of neurological impairment.

Keywords: spastic cerebral palsy, PET_positron emission tomography, brain metabolism, selective voluntary motor control, ankle motor task

\section{INTRODUCTION}

Children with spastic cerebral palsy $(\mathrm{CP})$ have developmental brain injuries primarily affecting the motor systems. Impairments of motor control are observed early in development (Fetters et al., 2004; Sargent et al., 2017) often preceding the detection of spasticity in children with CP. Deficits in gross motor function including mobility, strength, and balance are additional impairments. Spastic $\mathrm{CP}$ results from damage to the periventricular white matter containing descending motor tracts including the corticospinal tracts (CSTs) responsible for voluntary motor control (Bax et al., 2006; 
Volpe, 2009). White matter damage including the CSTs has been described and quantified in $\mathrm{CP}$ and correlated with motor and sensory function measures using magnetic resonance imaging (MRI) with diffusion tensor imaging (DTI) techniques (Hoon et al., 2009; Lee et al., 2011). While damage to the developing CSTs is a primary etiology in spastic CP, resulting compensatory adaptations have not been adequately studied relative to brain structure and activity, especially for lower extremity function in patients with bilateral involvement.

CSTs that originate in the motor cortex are responsible for skilled voluntary movement or selective motor control. The term "selective voluntary motor control" (SVMC) indicates the deliberate performance of isolated movements upon request (Fowler et al., 2010). Children with spastic CP and impaired SVMC may exhibit reduced speed of movement, mirror movements or abnormal reciprocal muscle activation patterns. Also, they are often unable to move their hip, knee and ankle joints in isolation, relying instead on closely coupled flexion and extension patterns to varying degrees (Fowler and Goldberg, 2009). In two studies, SVMC was more predictive of motor function than other aspects of CP (Østensjø et al., 2004; Voorman et al., 2007). Clinical measures of SVMC have been shown to correlate with mobility level (Fowler et al., 2009), gross motor function (Balzer et al., 2016; Noble et al., 2019) and gait (Fowler and Goldberg, 2009; Steele et al., 2015; Rha et al., 2016; Chruscikowski et al., 2017; Zhou et al., 2019).

Following a perinatal injury to the CSTs, alternative motor pathways develop that are forms of adaptive or maladaptive plasticity (Eyre, 2007; Friel et al., 2013; Gordon, 2016). This has been shown for animal models and the upper extremity of children with spastic hemiplegic CP. Ipsilateral CSTs from the uninvolved hemisphere can be preserved causing mirror movements and other impairments. In adults post stroke, it has been suggested that compensations by areas of the brain such as the rubrospinal tracts are utilized when damage to CSTs occur, producing synergistic flexor and extensor patterns in the involved extremities during voluntary movement (Yeo and Jang, 2010). While children with spastic CP sustain an early injury to the brain before the development of motor skills, similar compensatory pathways may be involved (Cahill-Rowley and Rose, 2014).

The structure of white matter tracts has been the focus of brain imaging studies in spastic CP (Scheck et al., 2012; Mailleux et al., 2020). Far less is known about neuromotor recruitment. Studies of brain activity during movement in $\mathrm{CP}$ have primarily focused on children with unilateral involvement during upper extremity fine motor or sensory tasks using functional magnetic resonance imaging (fMRI; Dinomais et al., 2013; Van de Winckel et al., 2013a,b). Only two fMRI studies investigating lower extremity movement in CP could be found (Phillips et al., 2007; Hilderley et al., 2018). All children were high functioning as they were able to walk and run independently (GMFCS level I) and could actively dorsiflex the ankle, which indicates a high level of selective movement. Despite these stringent inclusion criteria, excessive head movement during the motor task was problematic during fMRI data collection resulting in unusable data for some participants (Phillips et al., 2007) or limiting the number of available trials for analysis (Hilderley et al., 2018). Brain activation can also be studied using positron emission tomography (PET), a metabolic imaging technique that uses radioactive compounds to label functional brain metabolism (Phelps, 2000). A common biologically active molecule used is FDG, an analog of glucose. Regional glucose metabolism and accumulation represent the metabolic activity of the tissues (Alauddin, 2012). Radiotracer concentrations in specific regions of the brain are mapped on three-dimensional images of the brain that are reconstructed from MRI (Lee et al., 2007; Penny et al., 2011). Only one PET scan study could be found that investigated brain metabolism in $\mathrm{CP}$ (Lee et al., 2007) but it did not involve a motor task. An advantage of PET for evaluating neuromotor control is that motor task performance and tracer uptake occur before the imaging session. In contrast, the motor task occurs during imaging for fMRI requiring that head position be maintained during limb movement, excluding more children with $\mathrm{CP}$ from participation.

The purpose of this study was to examine the relationship between brain metabolism and SVMC in children with spastic bilateral CP during movement using PET. SVMC was evaluated using the Selective Control Assessment of the Lower Extremity (SCALE; Fowler et al., 2009). We hypothesized that a significant positive relationship between SCALE and the sensorimotor cortex would be found. Our secondary hypothesis was that significant correlations between SCALE and activation of other motor regions of the brain would be identified.

\section{MATERIALS AND METHODS}

\section{Participants}

Children with spastic bilateral CP were recruited from clinics and the community via mailings and flyers. This study was approved by the Human Subject Committee at the University of California, Los Angeles, CA, USA. Informed assents and consents were obtained from the participants and their guardians. Participants were recruited from the Center for $\mathrm{CP}$ at UCLA/OIC as well as the surrounding Los Angeles community. Data used for this analysis were collected as baseline information for a larger treatment intervention study. Inclusion criteria were: (1) age between 4 and 12 years; (2) diagnosis of spastic form of CP; and (3) ability to remain still for a minimum of $15 \mathrm{~min}$. Exclusion criteria were: (1) attention deficit or hyperactivity disorder; (2) seizure within the last 6 months; (3) participation in a research study that involves the use of radiation in the past 12 months; (4) fear of enclosed spaces, breathing or swallowing problems, dizziness, or fainting spells; (5) pacemaker, intrathecal baclofen pump or metal implants in the head or neck, other than tooth fillings; and (6) mechanical, cystic or other structural abnormalities on MRI. Following informed consent, each participant received a structural MRI. Participants who were unable to lie still or who had MRI exclusion factors were discontinued from study participation. A physical therapist assessed mobility using the Gross Motor Function Classification 
System (GMFCS; Palisano et al., 1997) and gross motor function using the Gross Motor Function Measure (GMFM; Bjornson et al., 1998).

\section{Selective Voluntary Motor Control Assessment}

Before PET scanning, SVMC was assessed by a physical therapist using SCALE, a validated and reliable clinical tool, which was developed for individuals with spastic CP (Fowler et al., 2009; Balzer et al., 2016). This assessment incorporates components of CST function including selectivity, reciprocation, and speed as well as the presence of involuntary movement at other joints including mirror movements of the contralateral extremity. Hip, knee, ankle, subtalar and toe joints are assessed using an isolated, reciprocal movement pattern and each joint is scored 0 (Unable), 1 (Impaired) or 2 (Normal). Scores are summed resulting in a possible score from 0 (absent SVMC) to 10 (normal SVMC) for each lower limb.

\section{PET Scan Data Acquisition}

PET scanning was used to acquire regional cerebral metabolic data. Participants were asked to fast for $3 \mathrm{~h}$ before the procedure to optimize glucose uptake. The process of injection and PET scan required approximately $2 \mathrm{~h}$ for completion. An intravenous line was placed and $185 \mathrm{MBq}$ (FDG) was administered using standard aseptic technique. A single small blood sample was obtained to establish the starting level of blood glucose. PET scans were performed 40 min post-FDG injection.

During this uptake period, the participant performed an ankle movement task with the lower extremity that demonstrated the least impairment. The task was limited to one lower extremity to examine regions of the brain that controlled movement for the ipsilateral vs. contralateral limb. The child was instructed by a physical therapist to perform a series of isolated ankle dorsiflexion and plantar flexion movements that were initiated every $2 \mathrm{~min}$ followed by a brief rest period. If movement occurred at other joints, verbal feedback was provided for initial attempts but the feedback was discontinued if the motion was obligatory.

\section{Whole Brain Analysis}

Relative quantification of regional brain activity was performed using NeuroQ ${ }^{\mathrm{TM}}$ (Syntermed Inc., Atlanta). This software corrects for tissue-based attenuation and then implements an algorithm for automatically measuring the number of radioactive events emitted by a positron source (gamma-ray lines of coincidence) per second detected by PET-scanner, emanating from pixel locations assigned by a computerized reconstruction algorithm. Statistical parametric mapping (SPM) methods were performed (Friston et al., 1995a,b) to co-register participant images and to reorient them into a standardized coordinate system using the SPM software package (Ashburner, 2009) from the Wellcome Department of Cognitive Neurology, Functional Imaging Laboratory (London, UK). Data were spatially smoothed and normalized to mean global activity as previously described (Silverman et al., 2011), except for a $12 \mathrm{~mm}$ (full-width half-maximum) smoothing filter that was applied to the images before statistical analysis. The set of pooled data were assessed with the t-statistic on a voxel-by-voxel basis, to identify the profile of voxels that significantly covaried with parameters characterizing each participant. To identify the anatomical label of the underlying voxel, we defined 240 standardized ROIs (sROIs) following the transformation of each PET scan to a template space (Tai et al., 1997) throughout the transaxial planes across the field of view. Normalized uptake values were determined for specific regions of the brain. Whole-brain voxel-wise Pearson correlations of metabolic activity vs. SCALE scores were performed. These results were correlated for multiple comparisons using cluster wise thresholding in SPM. All 240 sROIs and 47 volumes of interest (sVOIs) were used solely to obtain an anatomic parcellation of the brain and to identify the anatomical regions where there is cluster-wise statistical significance.

\section{Secondary ROI Analysis}

In the case of bilateral activation, it was necessary to further identify whether the FDG uptake was ipsilateral or contralateral to the moving limb. Therefore, a secondary ROI analysis was performed exclusively for those regions. Mean voxel activity for the bilateral ROIs was calculated following the transformation of each PET scan to a template space. This value was automatically normalized to the mean activity measured throughout that brain scan for each ROI and was used to correlate with SCALE. Importantly, for correlation analysis, only the anatomical ROI-based average instead of the statistical ROI-based average was used to avoid circular analysis.

\section{RESULTS}

Participant characteristics are shown for 10 children who were enrolled and underwent baseline testing in Table 1. The average age was 9 years, 4 months. Motor impairment ranged from mild (GMFCS Level II, GMFM 72.2, SCALE 7 bilaterally) to severe (GMFCS Level V, GMFM 36.0, SCALE scores $\leq 3$ ). Two participants exhibited bilateral SCALE score $=0$. Four children could walk and six used wheelchairs as their primary mode of mobility. One participant was dropped from the study after enrollment due to a significant structural abnormality that was identified on MRI. Of the remaining nine participants, eight had white matter damage including periventricular leukomalacia on MRI. Bilateral volume loss of the thalamus was additionally reported for one of these children.

During the ankle motor task, obligatory movement at other joints was observed in most participants. All six participants with low limb SCALE scores (0-2) were unable to isolate ankle motion and exhibited simultaneous hip and knee synergistic movement. The remaining three participants with limb scores of $\geq 5$ exhibited impaired SVMC at their ankle, due to an inability to perform at least $15^{\circ}$ of isolated ankle motion or the presence of movement at another joint. Mirror movement at the ankle was observed for one participant. 
TABLE 1 | Participant characteristics.

\begin{tabular}{llc}
\hline Demographics & & $\boldsymbol{n}=\mathbf{1 0}$ \\
\hline Age & Mean Age (SD) year, month & $9,4(1,4)$ \\
Gender & Age range years & $6-11$ \\
& Male & 6 \\
Ethnicity & Female & 4 \\
Race & Hispanic & 4 \\
& African American & 1 \\
GMFCS & Caucasian & 9 \\
& II & 2 \\
& III & 2 \\
CP Diagnosis-distribution & IV & 3 \\
& V & 3 \\
GMFM & Diplegia & 5 \\
SCALE & Quadriplegia & 4 \\
& Total body involvement & 1 \\
& Mean (SD) & $53.2(11.9)$ \\
& Left Mean (SD) & $2.7(2.5)$ \\
Sight Mean (SD) & $2.5(2.6)$ \\
\hline
\end{tabular}

SD, standard deviation; GMFCS, gross motor function classification system; GMFM, gross motor function measure; SCALE, selective control assessment of the lower extremity.

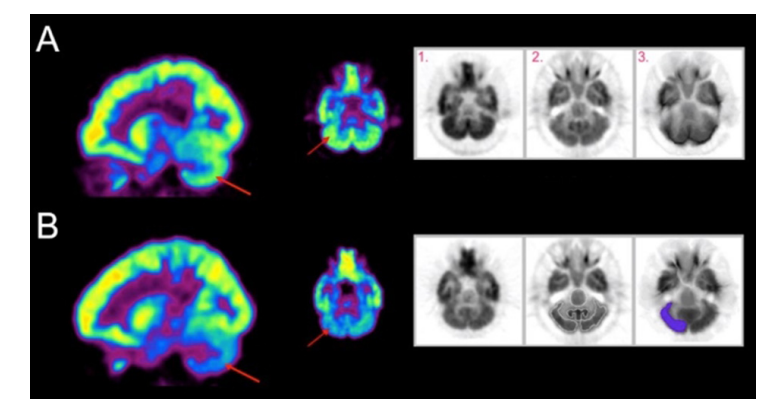

FIGURE 1 | Metabolic activity maps are shown for (A) an individual with a low Selective Control Assessment of the Lower Extremity (SCALE) score and (B) an individual with a high SCALE score. Red arrows indicate the right cerebellum. The gray scale images (inset) for each row show 1. Participant's original positron emission tomography (PET) scan, 2. Template PET scan on which standardized regions of interest are defined and 3 . Activity resampled on the template. Relative hypoactivity was found in the cerebellum of the individual with a high SCALE score, indicated by the indigo color using a rainbow scale (violet being the lowest and red being the highest).

\section{Qualitative Visualization of Whole Brain Metabolic Activity}

Exemplar metabolic activity maps for individuals with contrasting SCALE scores can be seen in Figure 1. The metabolic maps in the first two columns are color-coded (rainbow scale) showing normalized activity for sagittal and axial slices. A contrast between cerebellar activation levels for two participants with low vs. high SCALE scores can be seen.

\section{Whole-Brain Analysis}

The results of SPM analyses examining correlations between metabolic activity and SCALE scores are shown in Figure 2. The sensorimotor (SM) and superior parietal (sPL) cortex contralateral to the moving limb were significantly positively correlated with SCALE scores (SM: $t=8.06$, sPL: $t=6.70$; $p<0.0005)$. In contrast, a significant negative correlation

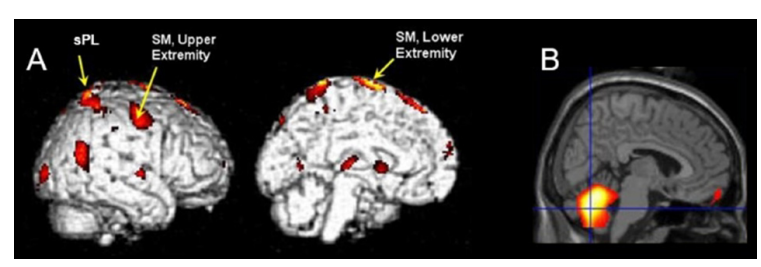

FIGURE 2 | (A) A three-dimensional volumetric rendering of significant positive correlations between SCALE and metabolic activity in the sensorimotor and the superior parietal cortex during movement of the contralateral limb shown on the lateral view and medial cross-section overlaid on an atlas ( $p<0.0005$, cluster corrected). (B) Voxels show significant negative correlations of metabolic activity with SCALE in the cerebellum ( $p<0.01$ cluster corrected) on the medial slice. Color indicates a significant relationship and yellow indicates a stronger relationship as compared to red. SPL, superior parietal lobe, SM, sensorimotor cortex.

of metabolic activity with SCALE was found in the entire cerebellum (peak $t=7.23, p<0.0005$ ). As both sides of the cerebellum were correlated with SCALE, the level of activation for the side ipsilateral vs. contralateral to the moving limb was not apparent from the whole-brain analysis. Therefore, a secondary analysis at the ROI level was performed.

\section{ROI Analysis}

We exclusively selected the cerebellum for further ROI analysis as it showed bilateral activation in the whole-brain analysis. Significant correlations between cerebellar ROI-averaged activity and SCALE score for the moving limb were found. As justified by tests of linearity, parametric Pearson correlation coefficients were used. A strong significant correlation was found between the SCALE scores for the moving limb and the ipsilateral cerebellum $(t=5.27, p<0.001$, Figure 3A). While the correlation between the activation level of the contralateral cerebellum and SCALE score was significant, the relationship was not as strong $(t=2.43$, $p=0.045$, Figure 3B).

\section{DISCUSSION}

This is the first study to document a relationship between impaired lower extremity SVMC and brain metabolic activity in children with CP. Previous researchers reported greater metabolism in bilateral motor and visual cortices and the cerebellum in children with spastic $\mathrm{CP}$ relative to typically developing children using PET; however, a motor task was not performed (Lee et al., 2007). As expected, children with higher levels of motor control in the present study exhibited greater activity in the cortical areas associated with motor function (sensorimotor and superior parietal cortices) contralateral to the moving limb. This finding is consistent with the primary motor cortex being the largest source of CSTs and the sensory cortex providing feedback during motor tasks. Further, the superior parietal cortex is known to be involved in adjusting posture and guiding movement of the limbs, particularly concerning visual-spatial perception and body awareness (Wolpert et al., 1998; Wolbers et al., 2003). A related finding from a fMRI study has shown activations in the primary motor and sensory areas 

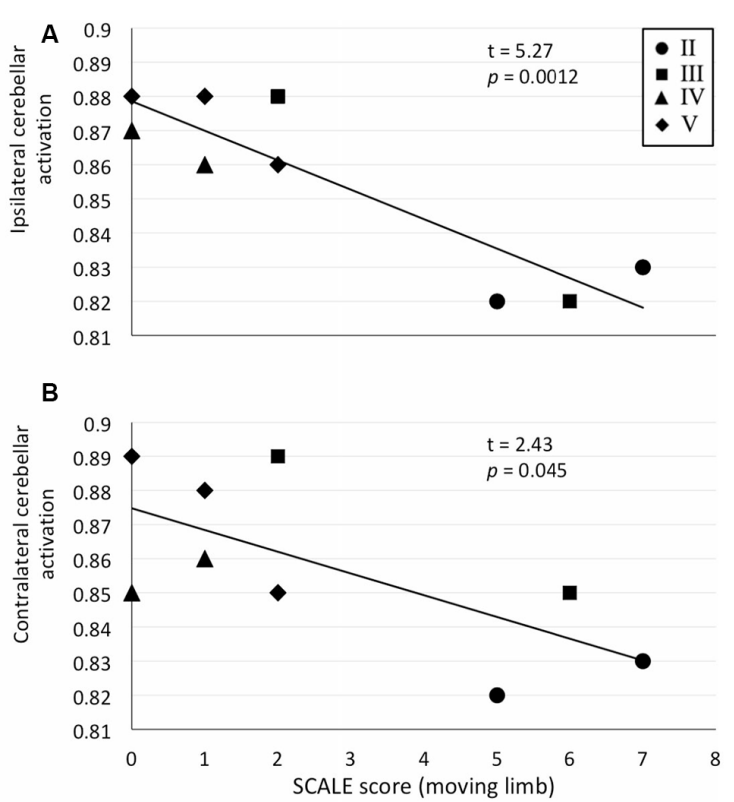

FIGURE 3 | Correlation plots for ROI-averaged cerebellar activation (normalized units) vs. SCALE score (from $0=$ absent to $10=$ normal) for the moving limb. (A) Ipsilateral cerebellum vs. SCALE score, $R^{2}=0.80$ and (B) Contralateral cerebellum vs. SCALE score, $R^{2}=0.46$. Gross Motor Function Classification System (GMFCS) level for each participant are indicated as II-IV.

in healthy adults performing a similar ankle dorsiflexion and plantarflexion task (Orr et al., 2008).

The sensorimotor region associated with the lower extremity exhibited a stronger correlation with SCALE than that associated with the upper extremity (Figure 2B). While activation of the sensorimotor cortex associated with the upper extremity would not be expected during ankle movement, the location of cortical activation has been found to vary for individuals with CP. Recently, motor evoked potentials with cortical stimulation were compared between adults with and without CP (Condliffe et al., 2019). Researchers reported that the "hotspots" for the soleus muscle of normal controls were in a tight cluster $2-3 \mathrm{~cm}$ lateral from the vertex (an anatomical landmark at the superior midpoint of the skull). For participants with $\mathrm{CP}$, however, the hot spots were farther away from the vertex, more dispersed and, in some cases more lateral. Some hot spots appeared to be closer to the typical atlas site for the upper as compared to the lower extremity.

A unique aspect of this brain activation study was the inclusion of children with absent or very low levels of SVMC for whom greater cerebellar activation was found. Historically, the role of the cerebellum in normal movement production has been attributed to the control of balance and coordination. Although the exact role of the cerebellum is not fully known, studies have shown that it is involved with motor learning and motor control (Manto et al., 2012). Authors of a consensus article concluded that the cerebellar motor systems consist of an intrinsically connected network involved in the optimization of movement performance during the early phases of motor learning. It may also contain internal feedback models associated with unconscious skilled movement (Manto et al., 2012). This may explain increased activation in our participants with poor SVMC for whom this motor task was novel and more challenging.

An alternative explanation for greater cerebellar activation in children with reduced SVMC may be recruitment via alternative pathways that communicate with the cerebellum during movement. Although isolated ankle motion was requested, children with low SCALE scores produced simultaneous hip and knee motion (abnormal obligatory synergies). Rubrospinal tracts, which originate in the red nucleus and communicate with the cerebellum, have been associated with these more primitive synergies (Cahill-Rowley and Rose, 2014). Major afferents project from the cerebellar and cerebral cortices to the red nucleus and the rubrospinal tract projects to cerebellar nuclei before reaching the spinal cord (Darras and Volpe, 2018). Animal studies have shown that when the cerebellar nuclei are stimulated, stereotyped motor synergies are produced (RispalPadel et al., 1982). This may explain why children with larger deficits in SVMC may have a greater reliance on the cerebellum resulting in the production of less skillful patterned movements when higher centers of motor control are impaired.

While a stronger relationship with SCALE was found for the ipsilateral cerebellum, significance was also found for the contralateral side. Typically, there is an ipsilateral association between the cerebellum and limb movement; however, bilateral activation during unilateral movement has been reported in normal controls and adults post-stroke (Cui et al., 2000; Ehrsson et al., 2002; Nair et al., 2003; Kapreli et al., 2006; Dong et al., 2007). Normal adults performing a finger tapping task primarily activated their ipsilateral cerebellum when using their dominant hand but activated their cerebellum bilaterally when using their non-dominant hand (Dong et al., 2007). In the same study, patients post-stroke recruited their contralateral as well as their ipsilateral cerebellum while performing the task with their involved hand. While our patient population had bilateral rather than unilateral limb motor impairment, these data support our findings. Children with CP with greater impairment exhibited higher levels of bilateral cerebellar activation than those with less impairment.

\section{Limitations}

This study had a small sample size but comparative fMRI studies contained even fewer participants with CP (Phillips et al., 2007; Hilderley et al., 2018). A control group of typically developing children was not studied. While the goal was to examine varying levels of brain metabolism based on selective motor control within $\mathrm{CP}$, knowledge of normal activation patterns under the same conditions is unknown. A common limitation in PET is the low anatomical resolution when mapping metabolic activity. Further, there may be smoothing of the metabolic maps when they are resampled to the SPM anatomical template, especially due to the lack of subject-specific MRI anatomical scan. However, this smoothing may also potentially lead to a gain in the signal to noise ratio locally over the image. Finally, due to statistical correction for a large number of voxel-wise multiple comparisons, there may be a loss of power although this loss 
is mitigated by both cluster-wise thresholding methods and a separate a priori ROI-based analysis.

\section{CONCLUSION}

In this study, we examined neuromotor control during an ankle motor task that was challenging for children with spastic $\mathrm{CP}$, particularly for those with low levels of motor control. As we hypothesized, there was a significant positive relationship between SVMC and metabolic activity in the sensorimotor cortex that was contralateral to the moving limb. Interestingly, we found that lower motor control was associated with greater cerebellar activation during the motor task. Decreased cortical and increased cerebellar activation in children with more impaired motor control may be related to task difficulty, activation of new motor learning paradigms in the cerebellum and potential engagement of alternative motor systems when CSTs are focally damaged. These results support SCALE as a clinical correlate of neurologic damage.

\section{DATA AVAILABILITY STATEMENT}

All datasets generated for this study are included in the article.

\section{REFERENCES}

Alauddin, M. M. (2012). Positron emission tomography (PET) imaging with ${ }^{18}$ F-based radiotracers. Am. J. Nucl. Med. Mol. Imaging 2, 55-76.

Ashburner, J. (2009). Computational anatomy with the SPM software. Magn. Reson. Imaging 27, 1163-1174. doi: 10.1016/j.mri.2009.01.006

Balzer, J., Marsico, P., Mitteregger, E., van der Linden, M. L., Mercer, T. H., and van Hedel, H. J. A. (2016). Construct validity and reliability of the selective control assessment of the lower extremity in children with cerebral palsy. Dev. Med. Child Neurol. 58, 167-172. doi: 10.1111/dmcn.12805

Bax, M., Tydeman, C., and Flodmark, O. (2006). Clinical and mri correlates of cerebral palsy: the european cerebral palsy study. JAMA 296, 1602-1608. doi: 10.1001/jama.296.13.1602

Bjornson, K. F., Graubert, C. S., Buford, V. L., and McLaughlin, J. (1998). Validity of the gross motor function measure. Pediatr. Phys. Ther. 10, 43-47. doi: 10.1097/00001577-199801020-00002

Cahill-Rowley, K., and Rose, J. (2014). Etiology of impaired selective motor control: emerging evidence and its implications for research and treatment in cerebral palsy. Dev. Med. Child Neurol. 56, 522-528. doi: 10.1111/dmcn. 12355

Chruscikowski, E., Fry, N. R. D., Noble, J. J., Gough, M., and Shortland, A. P. (2017). Selective motor control correlates with gait abnormality in children with cerebral palsy. Gait Posture 52, 107-109. doi: 10.1016/j.gaitpost.2016. 11.031

Condliffe, E. G., Jeffery, D. T., Emery, D. J., Treit, S., Beaulieu, C., and Gorassini, M. A. (2019). Full activation profiles and integrity of corticospinal pathways in adults with bilateral spastic cerebral palsy. Neurorehabil. Neural Repair 33, 59-69. doi: 10.1177/1545968318818898

Cui, S.-Z., Li, E.-Z., Zang, Y.-F., Weng, X.-C., Ivry, R., and Wang, J.-J. (2000). Both sides of human cerebellum involved in preparation and execution of sequential movements. Neuroreport 11, 3849-3853. doi: 10.1097/00001756-20001127000049

Darras, B. T., and Volpe, J. J. (2018). "Evaluation, special studies," in Volpe's Neurology of the Newborn, 6th Edition, eds J. Volpe, T. E. Inder, B. T. Darras, L. S. de Vries, A. J. du Plessis, J. J. Neil and J. M. Perlman (Philadephia, PA, USA: Elsevier), 861-873.

Dinomais, M., Lignon, G., Chinier, E., Richard, I., Ter Minassian, A., and Tich, S. N. T. (2013). Effect of observation of simple hand movement on brain

\section{ETHICS STATEMENT}

The studies involving human participants were reviewed and approved by Human Subjects Protection Committee, University of California, Los Angeles, USA. Written informed consent to participate in this study was provided by the participants' legal guardian.

\section{AUTHOR CONTRIBUTIONS}

$\mathrm{EF}, \mathrm{WO}, \mathrm{MG}$, and DS designed and conducted the experiments. $\mathrm{EF}, \mathrm{DS}$, and SJ analyzed the data. EF wrote the article. LS, SJ, and WO contributed to the discussion and edited the article.

\section{FUNDING}

This study was funded by a grant from the United Cerebral Palsy Research and Education Foundation \#R769-04.

\section{ACKNOWLEDGMENTS}

We thank the parents and children for their participation in this study.

activations in patients with unilateral cerebral palsy: an fMRI study. Res. Dev. Disabil. 34, 1928-1937. doi: 10.1016/j.ridd.2013.03.020

Dong, Y., Winstein, C. J., Albistegui-DuBois, R., and Dobkin, B. H. (2007). Evolution of FMRI activation in the perilesional primary motor cortex and cerebellum with rehabilitation training-related motor gains after stroke: a pilot study. Neurorehabil. Neural Repair 21, 412-428. doi: 10.1177/1545968306298598

Ehrsson, H. H., Kuhtz-Buschbeck, J. P., and Forssberg, H. (2002). Brain regions controlling nonsynergistic versus synergistic movement of the digits: a functional magnetic resonance imaging study. J. Neurosci. 22, 5074-5080. doi: 10.1523/JNEUROSCI.22-12-05074.2002

Eyre, J. (2007). Corticospinal tract development and its plasticity after perinatal injury. Neurosci. Biobehav. Rev. 31, 1136-1149. doi: 10.1016/j.neubiorev.2007. 05.011

Fetters, L., Chen, Y., Jonsdottir, J., and Tronick, E. Z. (2004). Kicking coordination captures differences between full-term and premature infants with white matter disorder. Hum. Mov. Sci. 22, 729-748. doi: 10.1016/j.humov. 2004.02.001

Fowler, E. G., Staudt, L. A., and Greenberg, M. B. (2010). Lower-extremity selective voluntary motor control in patients with spastic cerebral palsy: increased distal motor impairment. Dev. Med. Child Neurol. 52, 264-269. doi: 10.1111/j.14698749.2009.03586.x

Fowler, E. G., Staudt, L. A., Greenberg, M. B., and Oppenheim, W. L. (2009). Selective control assessment of the lower extremity (SCALE): development, validation, and interrater reliability of a clinical tool for patients with cerebral palsy. Dev. Med. Child Neurol. 51, 607-614. doi: 10.1111/j.1469-8749.2008. 03186.x

Fowler, E., and Goldberg, E. (2009). The effect of lower extremity selective voluntary motor control on interjoint coordination during gait in children with spastic diplegic cerebral palsy. Gait Posture 29, 102-107. doi: 10.1016/j.gaitpost. 2008.07.007

Friel, K. M., Chakrabarty, S., and Martin, J. H. (2013). Pathophysiological mechanisms of impaired limb use and repair strategies for motor systems after unilateral injury of the developing brain. Dev. Med. Child Neurol. 55, 27-31. doi: $10.1111 /$ dmcn. 12303

Friston, K. J., Ashburner, J., Frith, C. D., Poline, J.-B., Heather, J. D., and Frackowiak, R. S. (1995a). Spatial registration and normalization of images. Hum. Brain Mapp. 3, 165-189. doi: 10.1002/hbm.460030303 
Friston, K. J., Holmes, A. P., Poline, J. B., Grasby, P. J., Williams, S. C. R., Frackowiak, R. S., et al. (1995b). Analysis of fMRI time-series revisited. NeuroImage 2, 45-53. doi: 10.1006/nimg.1995.1007

Gordon, A. M. (2016). Impaired voluntary movement control and its rehabilitation in cerebral palsy. Adv. Exp. Med. Biol. 957, 291-311. doi: 10.1007/978-3-319-47313-0_16

Hilderley, A., Taylor, M., Fehlings, D., Chen, J., and Wright, F. (2018). Optimization of fMRI methods to determine laterality of cortical activation during ankle movements of children with unilateral cerebral palsy. Int. J. Dev. Neurosci. 66, 54-62. doi: 10.1016/j.ijdevneu.2018.01.004

Hoon, A. H. J., Stashinko, E. E., Nagae, L. M., Lin, D. D. M., Keller, J., Bastian, A., et al. (2009). Sensory and motor deficits in children with cerebral palsy born preterm correlate with diffusion tensor imaging abnormalities in thalamocortical pathways. Dev. Med. Child Neurol. 51, 697-704. doi: 10.1111/j. 1469-8749.2009.03306.x

Kapreli, E., Athanasopoulos, S., Papathanasiou, M., Van Hecke, P., Strimpakos, N., Gouliamos, A., et al. (2006). Lateralization of brain activity during lower limb joints movement. An fMRI study. NeuroImage 32, 1709-1721. doi: 10.1016/j. neuroimage.2006.05.043

Lee, J. D., Park, H.-J., Park, E. S., Kim, D. G., Rha, D.-W., Kim, E. Y., et al. (2007). Assessment of regional $\mathrm{GABA}_{A}$ receptor binding using $18 \mathrm{~F}$-fluoroflumazenil positron emission tomography in spastic type cerebral palsy. NeuroImage 34, 19-25. doi: 10.1016/j.neuroimage.2006.09.004

Lee, J. D., Park, H.-J., Park, E. S., Oh, M.-K., Park, B., Rha, D.-W., et al. (2011). Motor pathway injury in patients with periventricular leucomalacia and spastic diplegia. Brain 134, 1199-1210. doi: 10.1093/brain/awr021

Mailleux, L., Franki, I., Emsell, L., Peedima, M.-L., Fehrenbach, A., Feys, H., et al. (2020). The relationship between neuroimaging and motor outcome in children with cerebral palsy: a systematic review-Part B diffusion imaging and tractography. Res. Dev. Disabil. 97:103569. doi: 10.1016/j.ridd.2019. 103569

Manto, M., Bower, J. M., Conforto, A. B., Delgado-García, J. M., da Guarda, S. N. F., Gerwig, M., et al. (2012). Consensus paper: roles of the cerebellum in motor control-the diversity of ideas on cerebellar involvement in movement. Cerebellum 11, 457-487. doi: 10.1007/s12311-011-0331-9

Nair, D. G., Purcott, K. L., Fuchs, A., Steinberg, F., and Kelso, J. S. (2003). Cortical and cerebellar activity of the human brain during imagined and executed unimanual and bimanual action sequences: a functional MRI study. Cogn. Brain Res. 15, 250-260. doi: 10.1016/s0926-6410(02)00197-0

Noble, J. J., Gough, M., and Shortland, A. P. (2019). Selective motor control and gross motor function in bilateral spastic cerebral palsy. Dev. Med. Child Neurol. 61, 57-61. doi: 10.1111/dmcn.14024

Orr, E. L., Lacourse, M. G., Cohen, M. J., and Cramer, S. C. (2008). Cortical activation during executed, imagined and observed foot movements. Neuroreport 19, 625-630. doi: 10.1097/wnr.0b013e3282fbf9e0

Østensjø, S., Carlberg, E. B., and Vøllestad, N. K. (2004). Motor impairments in young children with cerebral palsy: relationship to gross motor function and everyday activities. Dev. Med. Child Neurol. 46, 580-589. doi: 10.1017/s0012162204000994

Palisano, R., Rosenbaum, P., Walter, S., Russell, D., Wood, E., and Galuppi, B. (1997). Development and reliability of a system to classify gross motor function in children with cerebral palsy. Dev. Med. Child Neurol. 39, 214-223. doi: 10.1111/j.1469-8749.1997.tb07414.x

Penny, W. D., Friston, K. J., Ashburner, J. T., Kiebel, S. J., and Nichols, T. E. (eds.) (2011). Statistical parametric mapping: the analysis of functional brain images. London: Academic Press, Elsevier.

Phelps, M. E. (2000). PET: the merging of biology and imaging into molecular imaging. J. Nucl. Med. 41, 661-681.

Phillips, J. P., Sullivan, K. J., Burtner, P. A., Caprihan, A., Provost, B., and Bernitsky-Beddingfield, A. (2007). Ankle dorsiflexion fMRI in children with cerebral palsy undergoing intensive body-weight-supported treadmill training: a pilot study. Dev. Med. Child Neurol. 49, 39-44. doi: 10.1017/s0012162207000102.x

Rha, D., Cahill-Rowley, K., Young, J., Torburn, L., Stephenson, K., and Rose, J. (2016). Biomechanical and clinical correlates of stance-phase knee flexion in persons with spastic cerebral palsy. PM\&R. 54, 11-18. doi: 10.1016/j.pmrj.2015.06.003

Rispal-Padel, L., Cicirata, F., and Pons, C. (1982). Cerebellar nuclear topography of simple and synergistic movements in the alert baboon (Papio papio). Exp. Brain Res. 47, 365-380. doi: 10.1007/bf00239355

Sargent, B., Reimann, H., Kubo, M., and Fetters, L. (2017). Infant intralimb coordination and torque production: influence of prematurity. Infant. Behav. Dev. 49, 129-140. doi: 10.1016/j.infbeh.2017.08.009

Scheck, S. M., Boyd, R. N., and Rose, S. E. (2012). New insights into the pathology of white matter tracts in cerebral palsy from diffusion magnetic resonance imaging: a systematic review. Dev. Med. Child Neurol. 54, 684-696. doi: 10.1111/j.1469-8749.2012.04332.x

Silverman, D. H., Geist, C. L., Kenna, H. A., Williams, K., Wroolie, T., Powers, B., et al. (2011). Differences in regional brain metabolism associated with specific formulations of hormone therapy in postmenopausal women at risk for AD. Psychoneuroendocrinology 36, 502-513. doi: 10.1016/j.psyneuen.2010. 08.002

Steele, K. M., Rozumalski, A., and Schwartz, M. H. (2015). Muscle synergies and complexity of neuromuscular control during gait in cerebral palsy. Dev. Med. Child. Neurol. 57, 1176-1182. doi: 10.1111/dmcn.12826

Tai, Y.-C., Lin, K. P., Hoh, C. K., Huang, S. H., and Hoffman, E. J. (1997). Utilization of 3-D elastic transformation in the registration of chest X-ray CT and whole body PET. IEEE Trans. Nuclear Sci. 44, 1606-1612. doi: 10.1109/23. 632740

Van de Winckel, A., Klingels, K., Bruyninckx, F., Wenderoth, N., Peeters, R., Sunaert, S., et al. (2013a). How does brain activation differ in children with unilateral cerebral palsy compared to typically developing children, during active and passive movements, and tactile stimulation? An fMRI study. Res. Dev. Disabil. 34, 183-197. doi: 10.1016/j.ridd.2012.07.030

Van de Winckel, A., Verheyden, G., Wenderoth, N., Peeters, R., Sunaert, S., Van Hecke, W., et al. (2013b). Does somatosensory discrimination activate different brain areas in children with unilateral cerebral palsy compared to typically developing children? An fMRI study. Res. Dev. Disabil. 34, 1710-1720. doi: 10.1016/j.ridd.2013.02.017

Volpe, J. J. (2009). Brain injury in premature infants: a complex amalgam of destructive and developmental disturbances. Lancet Neurol. 8, 110-124. doi: 10.1016/S1474-4422(08)70294-1

Voorman, J. M., Dallmeijer, A. J., Knol, D. L., Lankhorst, G. J., and Becher, J. G. (2007). Prospective longitudinal study of gross motor function in children with cerebral palsy. Arch. Phys. Med. Rehabil. 88, 871-876. doi: 10.1016/j.apmr.2007. 04.002

Wolbers, T., Weiller, C., and Büchel, C. (2003). Contralateral coding of imagined body parts in the superior parietal lobe. Cereb. Cortex 13, 392-399. doi: 10.1093/cercor/13.4.392

Wolpert, D. M., Goodbody, S. J., and Husain, M. (1998). Maintaining internal representations: the role of the human superior parietal lobe. Nat. Neurosci. 1, 529-533. doi: 10.1038/2245

Yeo, S. S., and Jang, S. H. (2010). Changes in red nucleus after pyramidal tract injury in patients with cerebral infarct. NeuroRehabilitation 27, 373-377. doi: 10.3233/nre-2010-0622

Zhou, J. Y., Lowe, E., Cahill-Rowley, K., Mahtani, G. B., Young, J. L., and Rose, J. (2019). Influence of impaired selective motor control on gait in children with cerebral palsy. J. Child. Orthop. 13, 73-81. doi: 10.1302/1863-2548.13.180013

Conflict of Interest: The authors declare that the research was conducted in the absence of any commercial or financial relationships that could be construed as a potential conflict of interest.

Copyright (c) 2020 Fowler, Oppenheim, Greenberg, Staudt, Joshi and Silverman. This is an open-access article distributed under the terms of the Creative Commons Attribution License (CC BY). The use, distribution or reproduction in other forums is permitted, provided the original author(s) and the copyright owner(s) are credited and that the original publication in this journal is cited, in accordance with accepted academic practice. No use, distribution or reproduction is permitted which does not comply with these terms. 